\title{
Propranolol reduces implicit negative racial bias
}

\author{
Sylvia Terbeck • Guy Kahane • Sarah McTavish • \\ Julian Savulescu • Philip J. Cowen • Miles Hewstone
}

Received: 3 December 2011 / Accepted: 1 February 2012 / Published online: 28 February 2012

(C) Springer-Verlag 2012

\begin{abstract}
Background Implicit negative attitudes towards other races are important in certain kinds of prejudicial social behaviour. Emotional mechanisms are thought to be involved in mediating implicit "outgroup" bias but there is little evidence concerning the underlying neurobiology. The aim of the present study was to examine the role of noradrenergic mechanisms in the generation of implicit racial attitudes.

Methods Healthy volunteers $(n=36)$ of white ethnic origin, received a single oral dose of the $\beta$-adrenoceptor antagonist, propranolol $(40 \mathrm{mg}$ ), in a randomised, double-blind, parallel group, placebo-controlled, design. Participants completed an explicit measure of prejudice and the racial implicit association test (IAT), 1-2 $\mathrm{h}$ after propranolol administration.

Results Relative to placebo, propranolol significantly lowered heart rate and abolished implicit racial bias, without affecting the measure of explicit racial prejudice. Propranolol did not affect subjective mood.
\end{abstract}

Electronic supplementary material The online version of this article (doi:10.1007/s00213-012-2657-5) contains supplementary material, which is available to authorized users.

S. Terbeck $(\triangle) \cdot$ M. Hewstone

Department of Experimental Psychology, University of Oxford,

South Parks Road,

Oxford OX1 3UD, UK

e-mail: sylvia.terbeck@psy.ox.ac.uk

G. Kahane $\cdot$ J. Savulescu

Oxford Uehiro Centre for Practical Ethics, University of Oxford,

Suite 8, Littlegate House, St Ebbes Street,

Oxford OX1 1PT, UK

S. McTavish • P. J. Cowen

Department of Psychiatry, Neurosciences Division,

University of Oxford, Warneford Hospital,

Oxford OX3 7JX, UK
Conclusions Our results indicate that $\beta$-adrenoceptors play a role in the expression of implicit racial attitudes suggesting that noradrenaline-related emotional mechanisms may mediate negative racial bias. Our findings may also have practical importance given that propranolol is a widely used drug. However, further studies will be needed to examine whether a similar effect can be demonstrated in the course of clinical treatment.

Keywords Noradrenaline · Propranolol $\cdot$ Implicit racial bias . Explicit prejudice

\section{Introduction}

Negative evaluations of minority groups are a central source of avoidance, discrimination, and conflict - problems which are of particular importance in today's increasingly cosmopolitan world. The dominance of political norms of equality and tolerance in Western democracies limits the overt expression of prejudice in such countries (Schuman et al. 1997). There is, however, extensive evidence that implicit bias - automatic negative attitudes associated with out-group members (Greenwald et al. 1998) — remains a live and a potent influence.

The implicit association test (IAT) (Greenwald et al. 2009) is a widely used method to assess implicit attitudes to which individuals may have limited introspective access (Nosek et al. 2007). In this item-sorting task, latency differences between prejudice congruent and incongruent trials are evaluated, to measure implicit bias. There is now extensive evidence that the IAT is a reliable measure of implicit attitudes towards social outgroups, whether based on race, sexual orientation, gender, or political preference (Nosek et al. 2007). IATs were developed precisely to reduce the effect of 
social desirability responses which might affect explicit prejudice responses (Greenwald et al. 1998). A recent metaanalysis further suggests that the IAT is a consistently better predictor of discrimination against out-group members than measures of explicit prejudice (Poehlman et al. 2004). It has been suggested, however, that implicit measures best predict subtle and spontaneous biased behaviour (Nosek et al. 2007), whereas explicit prejudice measures predict deliberate behaviour (Fazio et al. 1995).

Several studies have suggested that, compared to explicit prejudice, implicit prejudice involves a stronger emotional component. For example, Phelps et al (2000) found increased amygdala activity when white participants viewed faces of unknown black people, a finding confirmed by other studies (Amodio 2003; Lieberman et al. 2005; Wheeler and Fiske 2005). Importantly, the magnitude of amygdala activation was correlated with the participants' IAT score. However, these previous studies are largely correlational in nature, and cannot establish the causal mechanisms that underlie implicit bias.

Pharmacological interventions offer another way to study the causal role of emotional processes in implicit bias, and noradrenaline pathways involving $\beta$-adrenergic receptors are known to play a role in emotional memory and emotional perception (Cahill et al. 1994; Harmer et al. 2001) as judged by the effects of the non-selective $\beta$-adrenoceptor antagonist, propranolol. Besides its well-established effect on emotional memory via reduction in emotional conditioning (Chamberlaine et al. 2006), functional neuroimaging studies have also shown that propranolol leads to a reduction in amygdala responses to both facial expressions and visual emotional stimuli (Hurlemann et al. 2010a, b; van Stegeren et al. 2005).

In the present study, therefore we employed propranolol to test the hypothesis that emotional responses influenced by noradrenergic transmission play a mediating role in implicit but not in explicit forms of prejudice. We predicted that $\beta$-adrenoceptor blockade should lead to a reduction in implicit racial attitudes, as measured by the IAT, without a corresponding reduction in measures of explicit prejudice.

\section{Methods and materials}

\section{Participants}

We studied 36 healthy volunteers who gave full informed, written consent to the study. Participants were all of white ethnic origin, mostly from a British student population. They were screened by a trained clinician to be free of any Axis 1 Psychiatric Disorder using the Structured Clinical Interview for DSM-IV (SCID-IV); they also underwent a brief medical examination which included an ECG and blood pressure measurement to exclude those suffering any medical contraindication to propranolol administration (epilepsy, asthma, heart disease). Participants then completed the Beck Depression Inventory (BDI) (Beck et al. 1961) and the Eysenck Neuroticism Scale (Eysenck and Eysenck 1964). Ethical approval was obtained from the local NHS research ethics committee. Participants received $£ 30$ and travel expenses in compensation.

\section{Procedure}

Eligible participants were invited for a second visit to take part in the experiment. They were asked to refrain from alcohol and coffee on the study day. Participants were randomly allocated to take either propranolol ( $40 \mathrm{mg}$ orally) or placebo in identical capsules in a parallel group, doubleblind design. Pulse rate was monitored at 30-min intervals starting before drug administration and continuing until the end of the study. After drug treatment participants rested for $1 \mathrm{~h}$ after which the measure of explicit prejudice measure was administered $70 \mathrm{~min}$ and the IAT at $120 \mathrm{~min}$ after drug treatment; this timing was based on the pharmacokinetics of propranolol with peak plasma concentrations being reached between 1 and 2 h (Gilman 1996). Subjective mood was assessed using a Visual Analogue Scale (VAS), which asked participants to rate themselves on six emotions (tense, angry, happy, sad, tired, alert) on continuous scales ( $0-10$ anchors). Participants were instructed to report their "feeling at this moment". Participants completed the VAS scales at three time points: before, $1 \mathrm{~h}$ and $3 \mathrm{~h}$ after propranolol/placebo administration.

Tasks

\section{Explicit prejudice measure}

A feeling thermometer, an established tool for measuring explicit prejudice (Converse and Presser 1986), was used; on a 10-point scale, ranging from $0^{\circ}$ to $100^{\circ}$ (split into ten categories) - analogous to a thermometer - participants rated how "warm" they felt towards various groups. Besides white and black people, they also indicated their attitude towards homosexuals, Muslims, Christians, and drug addicts. Differences between the scales for in- versus outgroup ratings indicate the level of explicit prejudice.

\section{Implicit association test}

A computerised racial picture version of the IAT was used, administrated using e-prime software. The design of the task followed that of the original version used by Greenwald et al. (1998). 
The tasks consisted of seven blocks, each with 20 trials. Participants were asked to respond as quickly and accurately as possible. Participants were asked to perform a categorisation task by sorting positive/negative words and pictures of faces of black/white individuals. Target words appeared in the centre of the screen, categories on the left/right corner. To assign items to the left category participants pressed the "e" key, for the right category they pressed the "i" key. Blocks 1 and 5 contained positive/negative words, block 2 black/white faces, blocks 3, 4, 6 , and 7 combinations in randomised order (see also Table S1).

The IAT effect was determined by latency differences in the time to respond in the main trials: blocks 7 and 4 (e.g., prejudice congruent vs. prejudice incongruent blocks) as well as in the practice trials (blocks 3 and 6). The pictures of black and white faces, as well as positive and negative words, were taken from the original test.

\section{Statistics}

The IAT was analysed according to the improved algorithm as provided by Greenwald et al. (2003). In this method, the main trials (Blocks 4,7) as well as the practice trials (Blocks $3,6)$ are analysed. First, error latencies were replaced by the mean response time for correct trials $+600 \mathrm{~ms}$. Only data from blocks with less than $15 \%$ errors were analysed (one participant was excluded due to more than $20 \%$ errors on practice and main trials, for three other participants only the main trials were analysed due to too many errors in the practice trials). The two differences - between blocks 7 and 4 , and 6 and 3 - were divided by the pooled standard deviation for correct trials. The average of the corrected differences revealed the IAT effect.

The IAT, heart rate and VAS ratings were analysed using analysis of variance (ANOVA). For VAS and heart rate measures, there was one main between subject factor "treatment" (propranolol vs. placebo) and one within subject factor, "time". For the IAT the main between subject factor was also "Time" while the within-subject factor was "congruency" (congruent vs. incongruent) stimuli. Significant interactions on the ANOVA were followed up with posthoc unpaired $t$-tests (two-tailed). Scores on the explicit racial prejudice measure were analysed by subtracting the attitude score for white people from that for black people (the same procedure was used for the assessment of other explicit prejudices). Group differences were assessed with the Mann-Whitney $U$-test. Demographic data were analysed with unpaired $t$-tests (two-tailed).

\section{Results}

We studied 36 participants, 18 of whom received propranolol and 18 placebo. The groups were well matched in terms of demographic measures as well as measures of mood (Table 1). Relative to placebo, propranolol did not alter VAS ratings of any of the six emotions (no main or interaction effects on ANOVA, all $p>0.2$ ).

Propranolol significantly lowered heart rate (calculated as change from baseline) as shown by a significant main effect of treatment on the ANOVA $\left(F_{(4,30)}=6.56, p=0.02\right)$, as well as a time $\times$ treatment $\left(F_{(4,30)}=4.62, p=0.00\right)$. Post hoc $t$-tests revealed that the heart rate change (e.g., the drop of the pulse from baseline) was significantly greater in the propranolol group (at $60 \mathrm{~min}: M=-8.17, \mathrm{SD}=6.76$; at $90 \mathrm{~min}: M=-7.27, \mathrm{SD}=7.16$; at $120 \mathrm{~min}: M=-8.55$, $\mathrm{SD}=7.77$ ) as compared to the placebo group (at $60 \mathrm{~min}$ : $M=-3.18, \mathrm{SD}=4.74$; at $90 \mathrm{~min}: M=-.722, \mathrm{SD}=7.16$; at 120 min: $M=-1.67, \mathrm{SD}=9.19$ ) (at $60 \mathrm{~min}: \mathrm{t}(33)=2.51$, $p=0.02 ; d=-0.87$; at $90 \mathrm{~min}: t_{(34)}=2.4, p=0.02 ; d=0.78$; at 120 min: $t_{(34)}=2.4, p=0.02 ; d=0.8$ ).

We found no effect of propranolol on the measure of explicit prejudice as judged using the Mann-Whitney $U$-test for independent, non-parametric data. Additionally, no significant difference between the propranolol and placebo groups was found for religious prejudice, sexual prejudice, or prejudice against drug addicted individuals (all $p>0.2$ ), see Table 2.

The measure of the IAT reaction times showed the expected main effect of congruency $\left(F_{(1,33)}=13.60\right.$, $p=0.001$ ) (i.e., the IAT effect), as well as a significant congruency $\times$ treatment interaction $\left(F_{(1,33)}=6.20\right.$, $p=0.02)$. There was no main effect of treatment $\left(F_{(1,33)}=0.26, p=0.61\right)$. Post hoc $t$-tests revealed no significant difference between groups in mean response times (ms) on congruent (placebo: $M=702.98, \mathrm{SD}=86.67$; propranolol: $M=788.72, \mathrm{SD}=175.07$ ) or incongruent (placebo: $M=866.23, \mathrm{SD}=168.98$; propranolol: $M=820.50$, $\mathrm{SD}=106.68)$ trials $\left(t_{(33)}=-1.85, p>0.05 ; t_{(33)}=0.95\right.$, $p>0.05)$. However, an unpaired $t$-test showed the difference between congruent and incongruent trials (the IAT effect) was significantly smaller in the propranolol $(M=0.26$, $\mathrm{SD}=1.09)$ as compared to the placebo group $(M=1.02$, $\mathrm{SD}=0.84)\left(t_{(33)}=2.3, p=0.03 ; d=0.8\right)($ see Fig. 1$)$. Paired $t$-tests also revealed that an IAT effect (a significant difference between the congruent and incongruent trials) could be determined for both practice $(M=112.13, \mathrm{SD}=111.06)$ and main $(M=161.05, \mathrm{SD}=244.53)$ trials in the placebo group $\left(t_{(17)}=4.30, p=0.001 ; t_{(17)}=2.80, p=0.01\right)$, but not in the propranolol group $(M=-26.30, \mathrm{SD}=198.08 ; M=65.55$, $\left.\mathrm{SD}=153.10 ; t_{(18)}=-0.53, p>0.05 ; t_{(18)}=1.82, p>0.05\right)$.

Additionally, in the propranolol group $34 \%$ of the participants had a "negative" IAT score (they were faster in the "bias"-incongruent as compared to the congruent condition). In the placebo group no subject had such a score. This difference was significant $\left(\chi^{2}(1, n=37)=7.2\right.$, $p=0.02$ ). 
Table 1 Demographic data for propranolol and placebo group
$B D I$ Beck Depression Inventory, n.s. not significant

Non-significant differences: all $p>0.2$

\section{Discussion}

The main finding of our study is that propranolol significantly reduced implicit but not explicit racial bias. This supports our hypothesis that noradrenaline-mediated emotional responses play a role in the generation of implicit negative racial attitudes, and supports prior theorising suggesting a greater affective component in implicit attitudes (Stanley et al. 2008).

Propranolol in single doses is a well-established means of assessing the role of noradrenaline pathways in emotional learning and perception (Cahill et al. 1994; Harmer et al. 2001; Hurlemann et al. 2010a, b; Mihov et al. 2010). However, $\beta$-adrenoceptor antagonists have other neuropsychological effects which need to be considered in our findings. Our subjective ratings do not suggest that propranolol caused sedation relative to placebo. In addition, it seems unlikely that the observed effect on IAT was due to a general impairing effect of propranolol on cognitive performance, given that previous research has demonstrated that propranolol and other beta blockers do not impair performance on purely cognitive conflict tasks, such as the standard Stroop test (Harvey et al. 1977; Lasser et al. 1989; Nakano et al. 1978). In addition, propranolol did not cause an overall slowing of reaction time in the IAT task.
Further research is needed to clarify the causal mechanisms through which noradrenergic pathways may influence implicit bias. Although our study used only behavioural measures, prior studies have shown that propranolol can reduce amygdala response to visual stimuli associated with negative emotions such as fear and anger (Van Stegeren et al. 2005), and the role of noradrenergic transmission in the basolateral amygdala in emotional processing has also been demonstrated in animal studies (McGaugh 2000). The amygdala plays a key role in the early non-conscious appraisal of threat, and may therefore also be involved in the mediation of implicit racial prejudice. In line with this proposal, previous neuroimaging studies found that increased amygdala activation was associated with implicit prejudice but not with explicit racial attitudes (Phelps et al. 2000). However, Phelps et al (2003) found that a single patient with bilateral amygdala damage exhibited an intact IAT effect, leading the authors to suggest that the amygdala may not be a critical structure for the manifestation of implicit bias.

Phelps et al (2003) proposed instead that the amygdala may be involved in the acquisition of emotional bias, or that common neural pathways influence both behaviour on the IAT and responses in the amygdala to the task. It is also possible that in presence of amygdala damage, other compensatory mechanisms may be recruited in the IAT task. It
Table 2 Explicit Prejudice measure for propranolol and placebo group

Non-significant results: $p>0.2$

\begin{tabular}{llll}
\hline Group & Propanolol & Placebo & \\
\hline Explicit racial prejudice score & Mean rank $=18.22$ & Mean rank $=18.78$ & $\begin{array}{l}Z=-0.201 \\
\text { n.s. }\end{array}$ \\
Explicit religious prejudice score & Mean rank $=19.17$ & Mean rank $=17.83$ & $\begin{array}{l}Z=-0.399 \\
\text { n.s. }\end{array}$ \\
Explicit sexual prejudice score & Mean rank $=18.81$ & Mean rank $=18.19$ & $\begin{array}{l}Z=-0.195 \\
\text { n.s. }\end{array}$ \\
$\begin{array}{l}\text { Explicit Prejudice score: drug } \\
\text { addicted individuals }\end{array}$ & Mean rank $=16.58$ & Mean rank $=20.42$ & $Z=-0.1180$ \\
& & & n.s \\
\hline
\end{tabular}


Fig. 1 Mean response times in the IAT for both groups

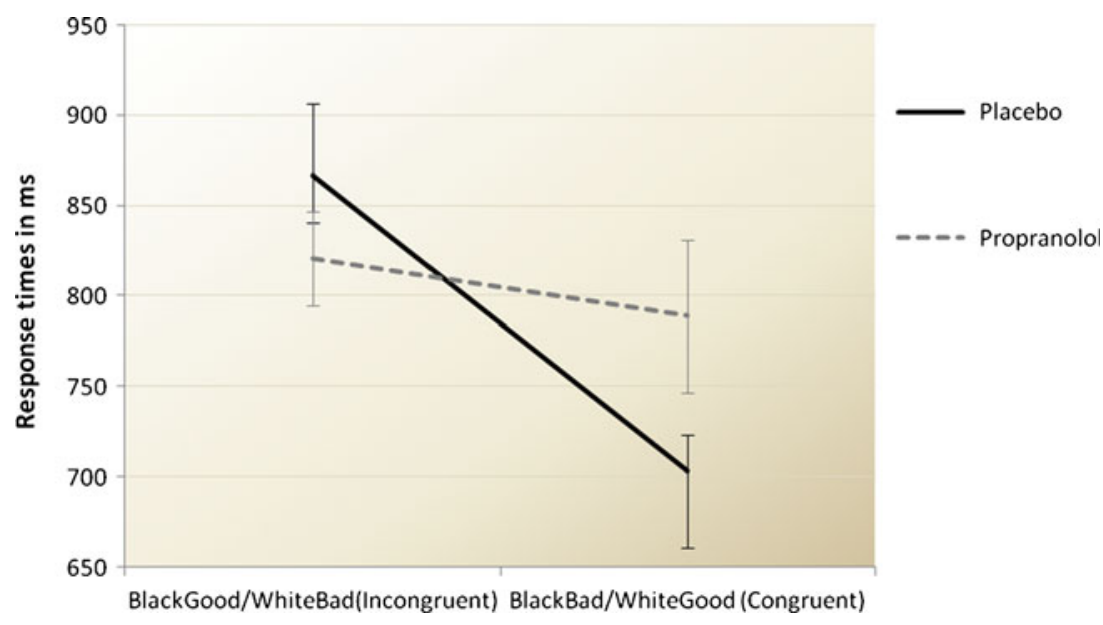

thus remains possible that automatic fear or threat responses related to amygdala activation and normally elicited by viewing of out-group faces might be the central emotional component mediating implicit attitudes. Propranolol might act at this level to produce its effect on the IAT, although further neuroimaging studies are needed to confirm reduction in limbic activation in response to such social cues following propranolol.

Another possibility is that the observed effect of propranolol on implicit attitudes is due to a decrease in peripheral sympathetic responses. It could be, for example, that negative implicit attitudes are triggered by somatic experiences dependent on increased sympathetic activation independent of amygdala activation. Propranolol may lead to a significant reduction in such response, and thereby reduce the "embodied" experience of emotional responses to a racial out-group. Further studies with a peripherally acting $\beta$-adrenoceptor antagonist, such as nadolol, are needed to determine whether the action of propranolol observed here is mediated by peripheral or central mechanisms. For example, Van Stegeren et al (1998) found that memory for emotional stimuli was impaired by propranolol but not nadolol, suggesting a central location for the relevant $\beta$-adrenoceptors.

The influence of propranolol on implicit attitudes observed in the present study may shed new light on the neurobiological mechanisms underlying implicit prejudice. Given the important role that implicit attitudes appear to play in overt forms of discrimination against out-group members, and the widespread use of propranolol for medical purposes, our findings might also be of practical interest, and require careful ethical consideration. Further research, however, is needed to clarify whether the observed effect would persist with sustained propranolol treatment, and whether propranolol can modulate implicit bias outside the laboratory.

It should be noted that our measures of explicit and implicit prejudice were given in a fixed order with a time gap between the two in order to try to avoid the tests influencing each other (e.g., to reduce awareness that "prejudice" was tested). This might have produced a confounding effect whereby previous explicit testing of prejudice interacted with propranolol to affect the IAT. It is also possible that the effect of propranolol to block $\beta$-adrenoceptors might have been more pronounced at the time of IAT testing. Against this latter possibility, however, was our finding that the physiological effect of propranolol (a significant drop in heart rate) was present and similar throughout the relevant testing period (60-120 min). This is in accordance with the peak plasma concentration for propranolol (Gilman 1996).

Recently, De Dreu et al (2011) reported that in-group favouritism was increased by oxytocin, a peptide hormone that has been implicated in trust, cooperation, and positive emotions. In one of their tasks, De Dreu et al (2011) used the IAT to measure the responses of Dutch participants to Arab or German minorities. The authors found, by comparing response times of positive associations made to in-group versus out-group members, that oxytocin increased response latency differences for positive associations, indicating an increase in in-group favouritism. This is of interest in the light of our findings because there are interactions between oxytocin and noradrenaline pathways, and in animals propranolol can lower oxytocin levels. Furthermore, Hurlemann et al. $(2010 \mathrm{a}, \mathrm{b})$ have suggested that the ability of oxytocin to facilitate socially reinforced learning is mediated via beta-adrenergic mechanisms in the amygdala. Further studies in humans are required to test this interesting proposal.

It is also possible that the effect of propranolol to decrease racial prejudice is mediated through a more general reduction in out-group denigration, in-group favouritism or both. Further studies could investigate these evaluative associations using, for example, the Go/No-go Association Task (GNAT; Nosek and Banaji 2001).

To conclude, the influence of propranolol on implicit attitudes observed in the present study may shed new light on the neurobiological mechanisms underlying implicit prejudice. Given the important role that implicit attitudes 
appear to play in overt forms of discrimination against outgroup members, and the widespread use of propranolol for medical purposes, our findings might also be of practical interest, and require careful ethical consideration. Further research, however, is needed to clarify whether the observed effect would persist with sustained propranolol treatment, and whether propranolol can modulate implicit bias outside the laboratory.

Acknowledgments We are grateful to the Wellcome Trust for funding part of this research (WT087208MF and WT086041MA).

Conflict of interest Professor Cowen has been a member of advisory boards of Eli Lilly, Servier and Lundbeck and has been a paid lecturer for Eli Lilly, Servier, Lundbeck and GlaxoSmithKline.

\section{References}

Amodio DM (2003) Individual differences in the activation and control of affective race bias as assessed by startle eyeblink response and self-report. J Pers Soc Psychol 84:738-753

Beck AT, Ward CH, Mendelson M, Mock J, Erbaugh J (1961) An inventory for measuring depression. Arch Gen Psychiatry 4:561-571

Cahill L, Prins B, Wber M, McGaugh JL (1994) Beta-adrenergic activation and emotional memory for events. Nature 371:702-204

Chamberlain SR, Mueller U, Blackwell AD, Robbins TW, Shakian BJ (2006) Noradrenergic modulation of working memory and emotional memory in humans. Psychopharmacology 188:397-407

Converse JM, Presser S (1986) Survey questions, handcrafting the standardized questionnaire, series: quantitative applications in the social science 63. Thousand Oaks, California: SAGE Publications

De Dreu CKW, Greer L, Gerben L, Van Kleef A, Shalvi S, Handgraaf MJJ (2011) Oxytocin promotes human ethnocentrism. Proc Natl Acad Sci 108:1262-1266

Eysenck HJ, Eysenck SBG (1964) Manual of the Eysenck personal inventory. Educational and Industrial Testing Service, San Diego

Fazio RH, Jackson JR, Dunton BC, Williams CJ (1995) Variability in automatic activation as an unobtrusive measure of racial attitudes: a bona fide pipeline? J Pers Soc Psychol 69:1013-1027

Gilman G (1996) The pharmacological basis of therapeutics, 9th edn. McGraw-Hill, New York

Greenwald AG, McGhee DE, Schwartz JLK (1998) Measuring individuals differences in implicit cognition: the implicit association test. J Per Soc Psychol 74:1464-1480

Greenwald AG, Banaji MR, Nosek BA (2003) Understanding and using the implicit association test: I. An improved scoring algorithm. J Pers Soc Psychol 2:197-216

Greenwald AG, Poelman TA, Uhlmann E, Banaji MR (2009) Understanding and Using the implicit association test: III. Meta-analysis of predictive validity. J Per Soc Psychol 97:17-41

Harmer CJ, Perrett DI, Cowen PJ, Goodwin GM (2001) Administration of beta-adrenoreceptor blocker propranolol impairs the processing of facial expressions of sadness. Psychopharmacology 154:383-389
Harvey PG, Clayton AB, Betts TA (1977) The effects of four antihypertensive agents on the Stroop colour-word test in normal male volunteer subjects. Psychopharmacology 54(2):133-138

Hurlemann R, Walter H, Rehme AK, Kukolja J, Santoro SC, Schmidt C, Schnell K, Musshoff F, Keysers C, Maier W, Kendrick KM, Onur OA (2010a) Human amygdala reactivity is diminished by the $\beta$-noradrenergic antagonist propranolol. Psychol Med 40:1839-1848

Hurlemann R, Patin A, Onur OA, Cohen MX, Baumgartner T, Metzler S, Dziobek I, Gallinat J, Wagner M, Maier W, Kendrick KM (2010b) Oxytocin enhances amygdala-dependent, socially reinforced learning and emotional empathy in humans. J Neurosci 30(14):4999-5007

Lasser WL, Nash J, Lasser V, Hamill S, Batey DM (1989) Effects of antihypertensive therapy on blood pressure control, cognition, and reactivity: a placebo-controlled comparison of prazosin, propranolol, and hydrochlorothiazide. Am J Med 86(1):98-103

Lieberman MD et al (2005) An fMRI investigation of race-related amygdala activity in African-American and Caucasian-American individuals. Nat Neurosci 8:720-722

McGaugh JL (2000) Memory-a century of consolidation. Science 287:248-251

Mihov Y, Mayer S, Musshoff F, Maier W, Kendrick KM, Hurlemann R (2010) Facilitation of learning by social-emotional feedback in humans is beta-noradrenergic dependent. Neuropsychologica 48:3168-3172

Narkano S, Hamp K, Hollister G (1978) Propranolol in experimentally induced stress. Psychopharmacology 59(3):279-284

Nosek BA, Banaji MR (2001) The go/no-go association task. Soc Cogn 19:625-666

Nosek BA, Greenwald AG, Banaji MR (2007) The implicit association test at age 7: a methodological and conceptual review. In Bargh JA (ed), Automatic processes in social thinking and behaviour. Psychology Press, (pp 265-292)

Phelps EA, O'Conner KJ, Cunningham WA, Funayama ES, Gatenby JC, Gore JC, Banaji MR (2000) Performance on indirect measures of race evaluation predicts amygdala activation. J Cogn Neurosci 12:729-738

Phelps EA, Cannistraci CJ, Cunningham WA (2003) Intact performance on an indirect measure of race bias following amygdala damage. Neuropsychologica 41:203-208

Poehlman TA, Uhlmann E, Greenwald AG, Banaji MR (2004) Understanding and using the implicit association test: III. Meta-analysis of predictive validity. Eur J Soc Psychol 38:922-934

Schuman H, Steeh C, Bobo L, Krysan M (1997) Racial attitudes in America: Trends and interpretations. Harvard University Press, Cambridge

Stanley D, Phelps E, Banaji M (2008) The neural basis of implicit attitudes. Curr Dir Psychol Sci 17:164

Van Stegeren AH, Everaerd W, Cahill L, McGaugh JL, Gooren LJG (1998) Memory for emotional events: Differential effects of centrally versus peripherally acting beta-blocking agents. Psychopharmacology 138:305-310

Van Stegeren AH, Goekoop R, Everaerd W, Scheltens P, Barkhof F, Kuijer JP, Rombouts SA (2005) Noradrenaline mediates amygdala activation in men and women during encoding of emotional material. NeuroImage 24:898-909

Wheeler ME, Fiske ST (2005) Controlling racial prejudice: social cognitive goals affect amygdala and stereotype activation. Psychol Sci 16:56-63 